\title{
VLTI - Early Results
}

\author{
Christoph Leinert \\ Max-Planck-Institut für Astronomie, Königstuhl 17, D-69117 \\ Heidelberg, Germany
}

\begin{abstract}
VLTI - the infrared interferometer integrated into the VLT complex on Paranal - is approaching operation as part of the scientific instrumentation of the VLTs, with the first call for proposals out for the observing period starting in spring 2004. Two first generation scientific instruments will become available: AMBER, working in the near-infrared and combining up to three telescope beams is scheduled to see first fringes and have commisioning early in 2004. MIDI, working in the $10 \mu \mathrm{m}$ band and combining two telescope beams, already is working in its basic observing mode. This contribution concentrates on a brief description of the latter instrument and the preliminary first scientific results obtained with it.
\end{abstract}

\section{Introduction}

From the beginning, the interferometric use of the VLTs has been part of the design of the Paranal observatory. Due to this solid infrastructure (Glindemann et al. 2003), already observations with the commissioning instrument VINCI (Kervella et al. 2003) and using in part the small, $40 \mathrm{~cm}$ diameter test siderostats, in part the speckled images of the $8.2 \mathrm{~m}$ unit telescopes (UTs), led to noteworthy results on the diameters of very low mass stars (Ségransan et al. 2003 ), on the diameters of our neighbouring stars $\alpha$ Cen and $\beta$ Cen (Kervella et al. 2003), on the flattening of the rapidly rotating star $\alpha$ Eri (de Souza et al. 2003), and on the innermost regions of the complex hot star $\eta$ Car (Paresce et al. 2003, van Boekel et al. 2003). The range of problems to be studied is greatly enhanced with the advent of the first generation instruments AMBER (nearinfrared, Petrov et al. 2003) and MIDI (mid-infrared, Leinert et al. 2003).

\section{The VLTI}

An overview on the VLTI site is shown in Figure 1. By now, all of the UTs can be used for interferometric measurements, providing baselines from $47 \mathrm{~m}$ to $130 \mathrm{~m}$. The results shown later refer to combining the light from UT1 and UT3 over a $\mathrm{B}=102 \mathrm{~m}$ baseline. At $10 \mu \mathrm{m}$ this means that structures of the size of 20 mas will be fully resolved. In the near-infrared, the resolution $\lambda / B$ over this typical baseline will be 2 mas - 5 mas. 


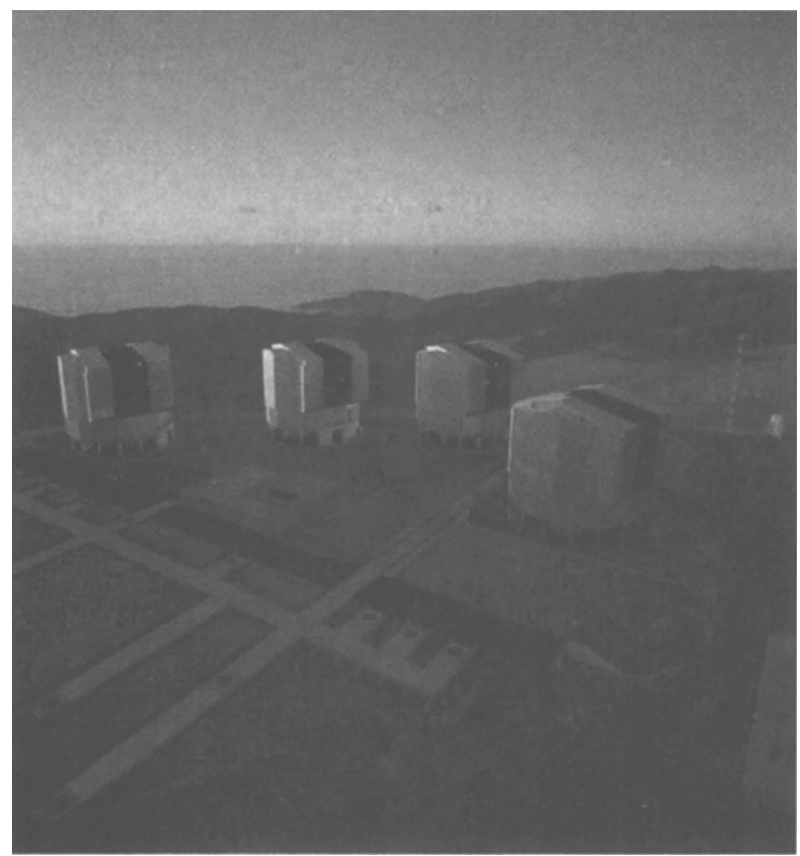

Figure 1. The four $8.2 \mathrm{~m}$ telescopes on Paranal, from left to right UT1, UT2, UT3, UT4. Also visible are the stations (holes) where the smaller $1.8 \mathrm{~m}$ auxiliary telescopes (ATs) can be positioned. The dark band running left-right shows the position of the underground delay line tunnel. (ESO PR Photo 14a/00)

\section{The near-infrared instrument AMBER}

This instrument will become available with two-beam combination in the first half of 2004 , and provide the possibility of three-beam combination and hence

Table 1. Expected sensitivities of AMBER on the UTs

a) for self-fringe tracking

Observing Mode

\begin{tabular}{lccc}
\hline High sensitivity $(50 \mathrm{~ms})$ & 10.5 & 11.6 & $\mathrm{~K}(\mathrm{mag})$ \\
High precision $(10 \mathrm{~ms})$ & 8.1 & 9.1 & 9.7 \\
\hline
\end{tabular}

$\mathrm{R}=35,1000,10000$ available as spectral resolution

b) with off-axis fringe tracking, $\mathrm{S} / \mathrm{N}=5$ in $4 \mathrm{~h}$

\begin{tabular}{lccc} 
Spectral resolution & $\mathrm{J}(\mathrm{mag})$ & $\mathrm{H}(\mathrm{mag})$ & $\mathrm{K}(\mathrm{mag})$ \\
\hline $\mathrm{R}=35$ (low) & 18.7 & 20.0 & 19.4 \\
$\mathrm{R}=1000$ (intermediate) & 15.8 & 17.1 & 17.4 \\
$\mathrm{R}=10000$ (high) & 13.3 & 14.6 & 15.1 \\
\hline
\end{tabular}


closure phase determination the year after. The expected sensitivities shown in Table 1 are very encouraging and promise access to interferometric studies of wide classes of objects. The sensitivities given in the table are given for the case of self-fringe tracking, available from the beginning, and for off-axis fringe tracking, an option to be introduced at the VLTI after 2005. The sensitivities refer to combining the light of $8.2 \mathrm{~m}$ UT telescopes. The limiting sensitivities for use of the $1.8 \mathrm{~m}$ ATs are lower by $3.1 \mathrm{mag}$.

\section{The mid-infrared instrument MIDI}

The principle of measurements can be followed in Figure 2. In front of the dewar, the beams from the two telescope each pass a small instrument-internal delay line, made up of four plane mirrors, of which the two forming a back-reflecting corner can be moved by a piezo device. During observations, this device is used to rapidly, within a few $100 \mathrm{~ms}$, step the optical path difference between the two beams over a few wavelengths. This leads to a temporal modulation of the order of $20 \mathrm{~Hz}$ with opposite phase for the two interferometric output beams,

Principle of MIDI - the Mid-Infrared Interferometer for the VLTI

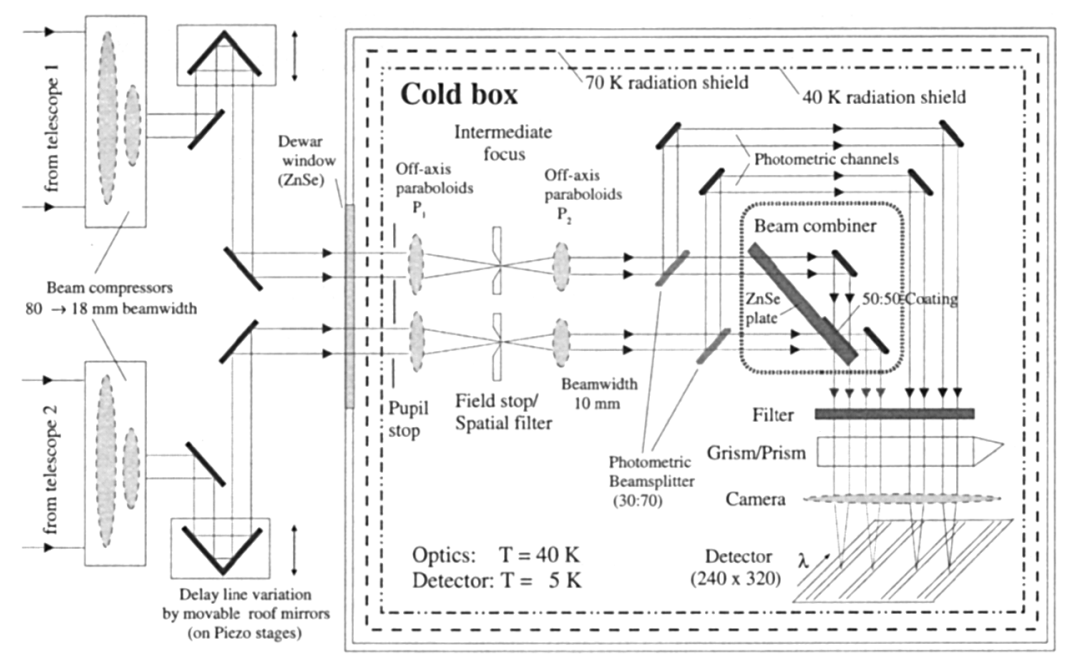

Figure 2. Optical light path through MIDI. The beams from two telescopes are superimposed and combined "on axis", i.e. parallel and concentric, on the $50 \% / 50 \%$ coating on the back of the beamcombiner plate. This is close to pupil plane. From the two interferometric beams leaving the beamcombiner, two images, resp. two spectra - if a prism or grism is inserted - are formed on the detector, representing the interferometric signal at opposite phases. For calibration purposes, a fraction of the incoming light beams could be extracted before entering the beam combiner, which would lead to an additional two images or spectra on the detector. 
the fringe amplitude of which measures the correlated flux. Because of the high thermal background radiation at $10 \mu \mathrm{m}$ and after 28 warm optical elements, we determine from the detected signal, more precisely from the difference of the two interferometric outputs, the correlated flux only. The brightness of the source is determined by separate photometric measurements where the background is removed by chopping. The detector is a Raethyon Si:As impurity band conduction (IBC) detector with $320 \times 240$ pixels.

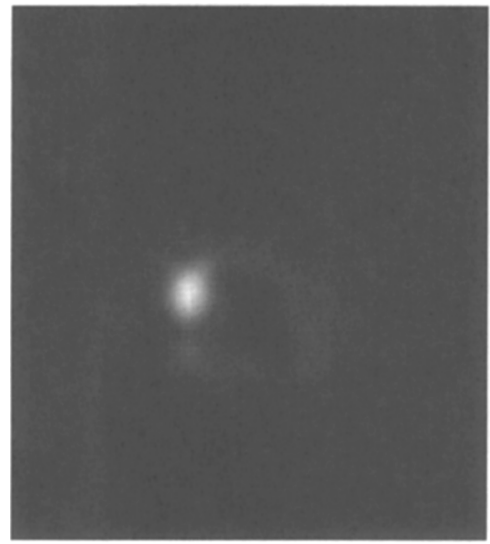

chopped raw data

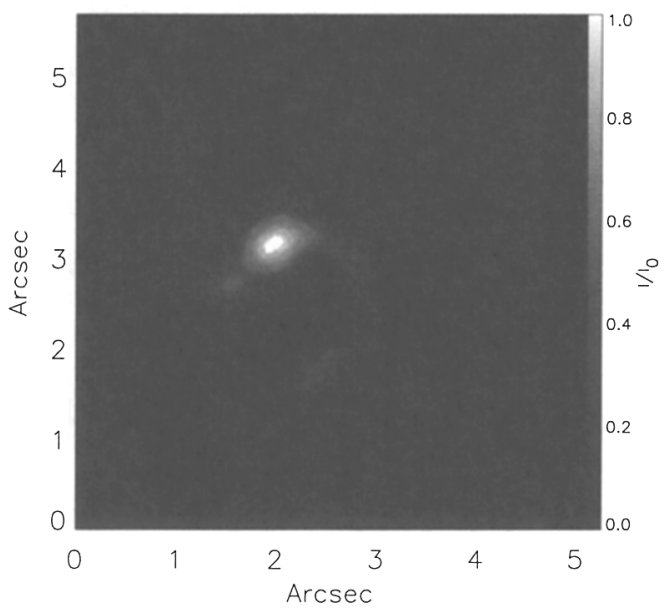

deconvolved

Figure 3. Eta Car in the $2^{\prime \prime}$ diameter interferometric field of MIDI. The raw data are as taken during acquisition through a filter at $8.7 \mu \mathrm{m}$, north is here upper left at a position angle of $\approx 30^{\circ}$, while the deconvolved data are in standard orientation with $\mathrm{N}$ up. Contrast has been enhanced by plotting signal ${ }^{1 / 4}$.

MIDI offers the choice of several focal plane diaphragms, of several filters and of spectral resolution by a prism $(\mathrm{R} \approx 30)$ or a grism $(\mathrm{R} \approx 230)$. At this time and for the measurements shown here, the interferometric observations have been restricted to use of a $0.5^{\prime \prime}$ wide long slit and use of the prism.

It should be emphasised that MIDI on the VLTI has a nonzero interferometric field. Although its diameter of $2^{\prime \prime}$ may appear almost negligible, it is of invaluable help for centering the object and, during acquisition, automatically delivers $\mathrm{N}$ band images of the object under study containing sometimes great detail and new information (see Figure 3). Similarly, for the present observations with the prism, the photometric measurement necessary to calibrate the observed fringe amplitudes automatically give $\mathrm{N}$ band spectra of good quality. The object in Figure 4, e.g., shows a strong silicate emission feature, the shape of which corresponds to a larger particle size than typical for the insterstellar medium. 

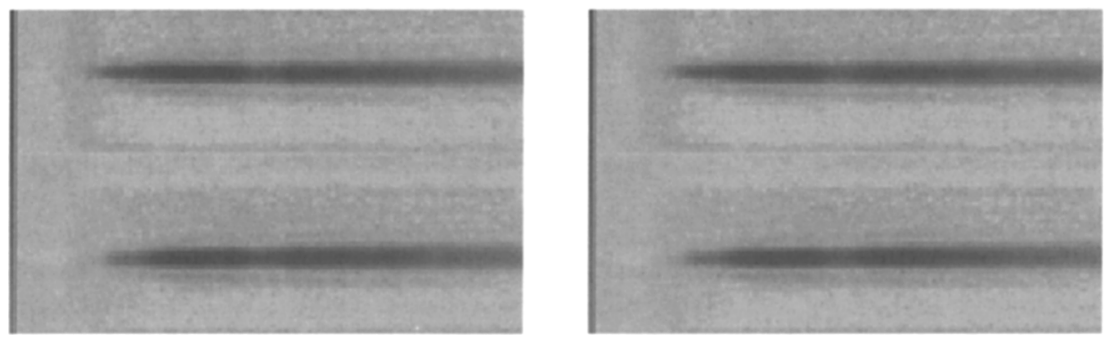

Figure 4. Spectrum of the Herbig Ae/Be star KK Oph. Left: the two traces on the detector as obtained during a non-interferometric exposure taken for photometric calibration from the two telescope beams. Wavelength is increasing towards the right. Right: raw counts (broken line) and calibrated flux resulting from these traces. Note the depression due to the atmospheric ozone band in the raw data.

\section{First scientific results with MIDI}

The first scientific run of the $10 \mu \mathrm{m}$ interferometer MIDI on the VLTI happened from June 11 - 16, 2003. The programme included observations of young stars, evolved stars and a first attempt to detect and resolve mid-infrared emission from the nucleus of an active galaxy. The telescopes UT1 and UT3 available for these measurements provided a baseline of $102 \mathrm{~m}$ which, depending on the position of the source on the sky, got projected to smaller effective values of down to below $80 \mathrm{~m}$. The images of stars brighter than $\mathrm{V}=14 \mathrm{mag}$ were stabilised in tip/tilt in the Coudé foci of the two telescopes. Seeing was good to very good $\left(<0.5^{\prime \prime}\right)$ during these days and the sky mostly clear, resulting in reliable measurements. The measurement sequence consisted of three parts: image taken during acquisition, interferometric scans with light spectrally dispersed by prism, chopped exposures of the prism spectrum separately for the two telescopes. This sequence was repeated for the interferometric calibrator stars. The time neede for such an object/calibrator pair was 1 hour. In the following, the emphasis will be on the results on young stars.

Herbig Ae/Be stars. In the last years, modelling of the dusty environment of Herbig $\mathrm{Ae} / \mathrm{Be}$ stars has concentrated on reproducing the observed spectral energy distributions (SEDs) over a large wavelength range from optical to far infrared and sub-mm wavelengths. The models of Dullemond et al (2001) and Dominik et al. (2003) treat passive disk models in which the inner rim of the dust distribution is puffed up by the overproportional heating it receives due to the unattenuated exposure to the stellar radiation. This leads to shadowing effects on the disk and allows to explain the sources with strong mid-infrared excess as those having a flaring disk geometry where much of the dusty material gets out of the shadow of the inner rim, while this is not the case for the sources with lesser mid-infrared excess. Calculating the spatial distribution of emitted infrared radiation in the $\mathrm{N}$ band, visibility values of the order of $10 \%$ 


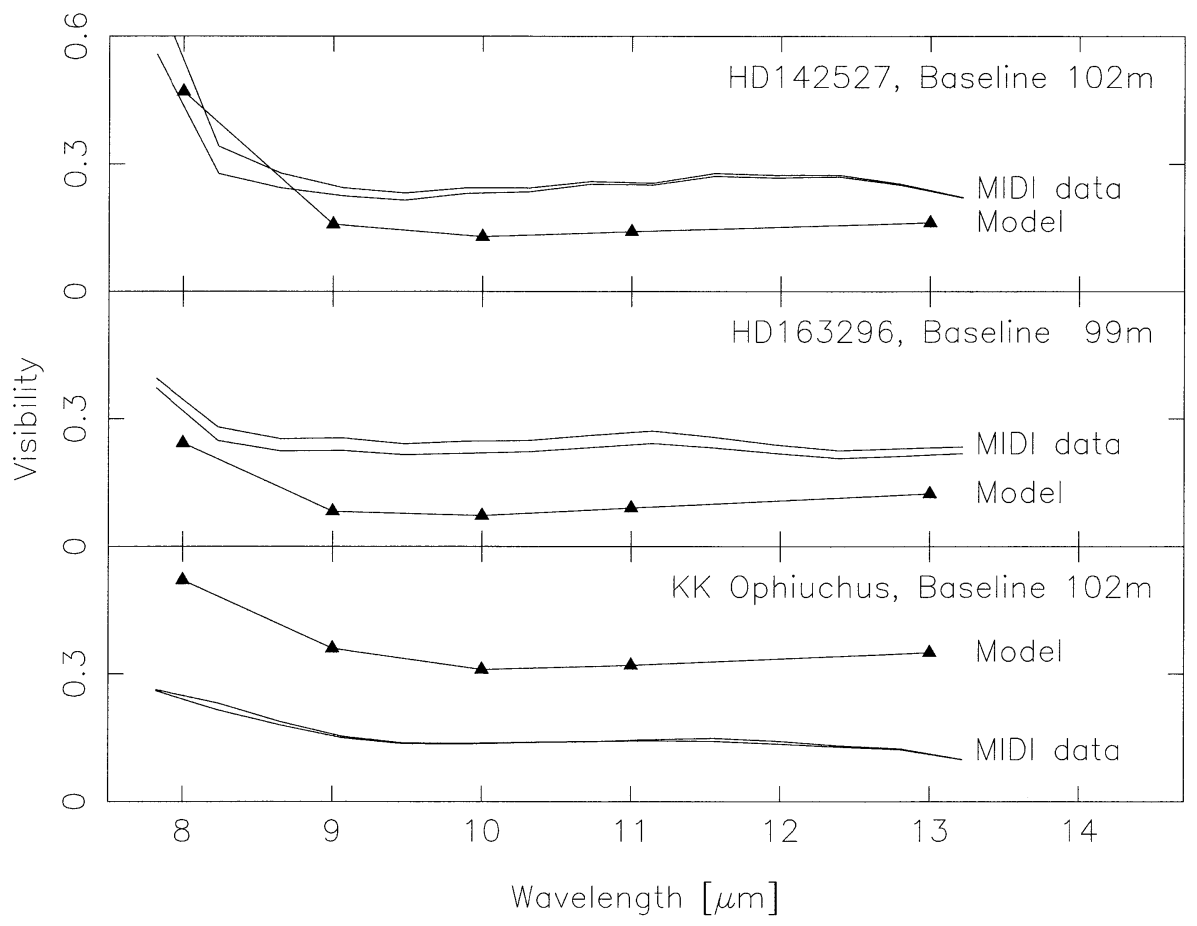

Figure 5. Comparison of measured wavelength-dependent visibilities with predictions from models fitting the spectral energy distributions of these Herbig Ae/Be stars. HD 142527 is classified as a group I source, modelled by a flaring disk, while HD 163296 and KK Oph are group II sources lacking the corresponding strength of mid-infrared enhancement.

are predicted, with little wavelength dependence except for a marked increase at the shortest wavelengths of the $10 \mu \mathrm{m}$ window. While single-telescope observations do not have sufficient spatial resolution to probe the relevant size scales of several AUs at the stars, the interferometric measurements of MIDI promised to offer a direct geometrical check on these models.

Figure 5 shows the results obtained with MIDI for three of these objects (double lines because of using two different point-like sources for calibration of each of the interferometric measurements) compared to the model predictions. While the general trend with wavelength predicted by the models is reproduced, the absolute level of the visibilities, which means the angular size of the emitting regions is not very well confirmd; it may be off by $30 \%-50 \%$, and this apparent discrepancy has to be further studied. But the important point is that indeed MIDI on the VLTI has the right sensitivity and spatial resolution to study the circumstellar disks of the closer-by Herbig Ae/Be stars.

T Tauri stars. For the T Tauri stars, because of their lower luminosity, thermal $10 \mu \mathrm{m}$ emission from circumstellar material will come from regions 


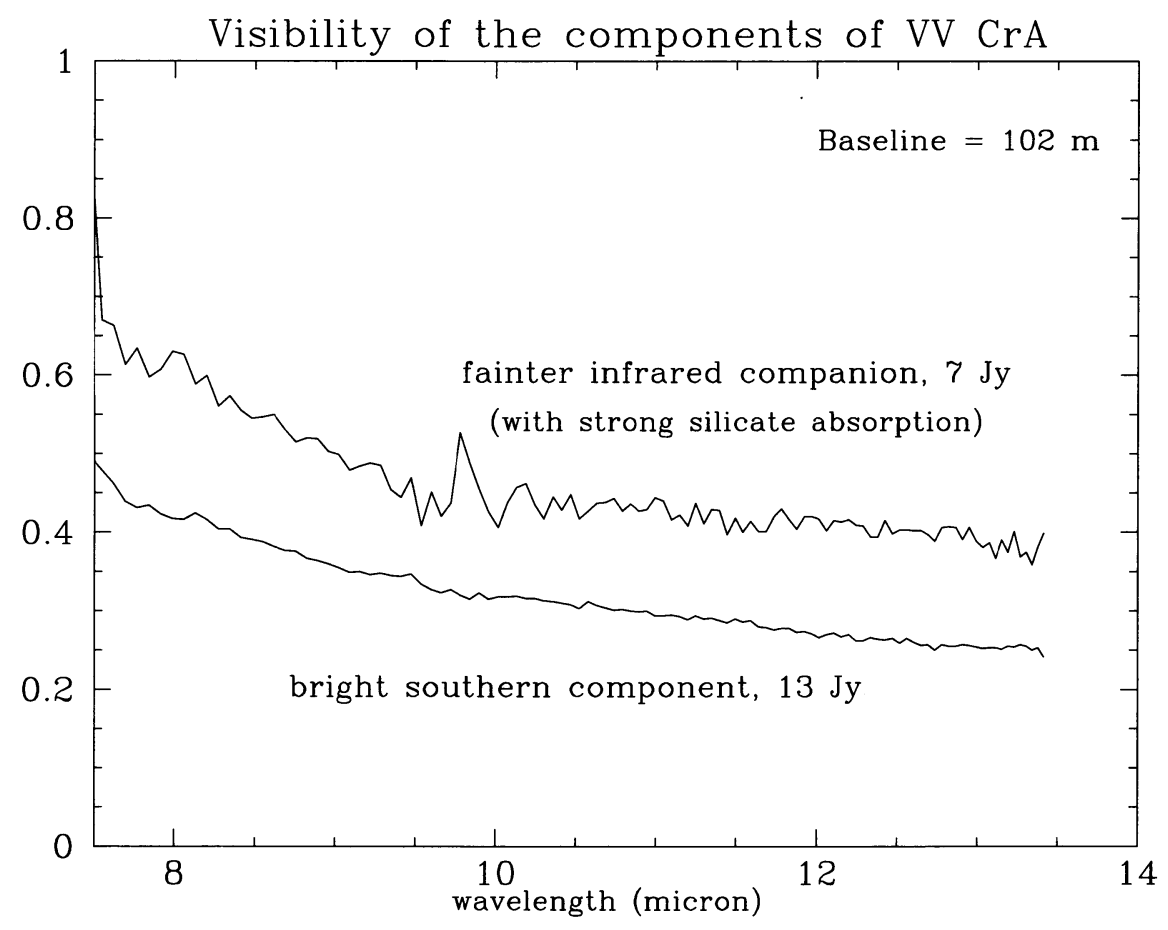

Figure 6. Spectrally resolved visibilities for the two components of the wide $\left(2.1^{\prime \prime}\right.$ separation) T Tauri binary VV CrA.

closer to the star than in the case of Herbig Ae/Be stars, and it is therefore less certain that MIDI on the VLTI will be able to resolve these circumstellar disks. The result of first observations on the binary T Tauri star VV CrA, one of the brighter ones of this group, is shown in Figure 6. The circumstellar thermal emission could be resolved for both components separately. The visibility profiles and hence distribution of emission for different wavelengths looks similar among the components and also similar to what was found for the Herbig Ae/Be stars (Figure 5). Apparently, the brighter southern component has the more extended (lower visibility) circumstellar thermal emission. To demonstrate that the emission in these cases does come from a flattened, disk-like structure will require several additional measurements at different baseline (spatial resolution) settings and for different position angles of the projected baseline with respect to the source. This should be feasible with the present instrument setup on the VLTI.

\section{Outlook}

In its present self-fringe tracking configuration, MIDI on the VLTI is able to interferometrically observe sources where the compact structures, unresolvable on single-dish observations have a $10 \mu \mathrm{m}$ flux of $>2 \mathrm{Jy}$. This allows a wealth 
of studies on young stars, is by far sufficient for the usually much brighter AGB sources, and even allowed first observations on the nucleus of NGC 1068. Gradual improvements are expected from actions planned for the coming months: perfecting the transmission of the VLTI delay lines, introducing a tip/tilt sensor close to the beam-combining instruments MIDI and AMBER. Large steps ahed in the range of possible observations are expected from the introduction of external fringe tracking (VLTI project FINITO, Gai et al. 2003), which should allow MIDI to go to at least 10 times fainter sources by blindly adding up the stabilised interferometric fringe patterns, as well as by introduction of the smaller $1.8 \mathrm{~m}$ auxiliary telescopes. These will be useful in the case of MIDI for the brighter sources only, but will offer a much improved coverage of baseline orientation and much improved availability of observing time for interferometric observations. These improvements, both planned for 2004, will allow to study more complex spatial distributions. Off axis fringe tracking, imaging and high precision differential astrometry are planned to follow (VLTI project PRIMA, Delplancke et al. 2003) and should make the VLTI with its instruments MIDI and AMBER a very versatile instrument for high spatial resolution astronomy.

Acknowledgments. Very many people have contributed to make possible the first observations with the $10 \mu \mathrm{m}$ instrument MIDI, both on the side of the instrument team and on the side of ESO for the infrastructure of the VLTI. Listing here those officially representing the instrument: Uwe Graser, Rens Waters, Guy Perrin, Annelie Glazenborg-Kluttig, Walter Jaffe, Bruno Lopez and officially connected to MIDI at ESO: Markus Schoeller, Andrea Richichi, S'ebastien Morel, Peter Biereichel, Andreas Glindemann, Francesco Paresce and those directly contributing to the paper: Olivier Chesneau, Rainer Köhler, Roy van Boekel, Frank Przygodda only means that the same sincere thanks also go to all the others of the two teams for their help and manyfold contributions.

\section{References}

de Souza, A.D., Jr. et al., 2003, A\&A, in press

Dominik, C., Dullemond, C.P., Waters, L.B.F.M., \& Walch, S., 2003, A\&A, 398, 607

Dullemond, C.P., Dominik, C., \& Natta, A., 2001, ApJ, 560, 957

Gai, M.et al., 2003, ESO conference and workshop proceedings 58, eds. E. Vernet, R. Ragazzoni, S. Esposito \& N. Hubin, p. 329

Glindemann, A. et al., 2003, SPIE, 4838, 89

Kervella, P. et al., 2003, SPIE, 4838, 858

Kervella, P. et al., 2003, A\&A, 404, 1087

Leinert, C. et al., 2003, SPIE, 4838, 893

Ségransan, D., Kervella, P.,Forveille, T , \& Queloz, D., 2003, A\&A, 397, L5

Paresce, F. et al., 2003, SPIE, 4838, 235

Delplancke, F. et al., 2003, IAU Symposium 221, this volume

Petrov, R., 2003, SPIE, 4838, 924

van Boekel, R. et al., 2003, A\&A Letter, in press 